\title{
ANÁLISE DO ABSENTEÍSMO E PERFIL DE USUÁRIO DE UM SERVIÇO DE RADIOLOGIA EM UMA FACULDADE DE ODONTOLOGIA PÚBLICA
}

\author{
ABSENTEEISM AND USER PROFILE ANALYSIS OF A RADIOLOGY SERVICE ON A PUBLIC \\ DENTISTRY SCHOOL
}

\author{
Nádia Assein ARÚS 1; Kelly Ahmad Pinheiro de LIMA 2; Priscila Fernanda da \\ SILVEIRA ${ }^{1 ;}$ Mariana Boessio VIZZOTTO ${ }^{1 ;}$ Heraldo Luis Dias da SILVEIRA ${ }^{1}$
}

\author{
1 Professor do Departamento de Cirurgia e Ortopedia da \\ Faculdade de Odontologia da Universidade Federal do Rio \\ Grande do Sul \\ 2 Cirurgiã dentista graduada pela Faculdade de \\ Odontologia da Universidade Federal do Rio Grande do \\ Sul \\ Correspondência: \\ Nome: Priscila Fernanda da Silveira \\ Email: prifsilveira@ufrgs.br \\ Endereço: Faculdade de Odontologia - UFRGS Ramiro \\ Barcelos, 2492 - Bairro Santana- Porto Alegre- CEP \\ 90035-003
}

\section{RESUMO}

Introdução: O Serviço de Radiologia da Faculdade de Odontologia da Universidade Federal do Rio Grande do Sul (FO-UFRGS) atende as necessidades de pacientes oriundos do Sistema Único de Saúde (SUS), realizando radiografias odontológicas, as quais são relevantes para o diagnóstico e planejamento clínico. A listagem do agendamento desses exames constitui um banco de dados com potencial de gerar importantes indicadores para o aprimoramento do serviço à população. Objetivo: Avaliar o perfil dos pacientes, número e origem dos encaminhamentos, e o absenteísmo frente aos agendamentos de exames radiográficos do Serviço de Radiologia da FOUFRGS. Materiais e métodos: Os dados foram coletados via Sistema de Regulação (SISREG) da Secretaria Municipal de Saúde de Porto Alegre (SMS-POA) e foram analisadas as seguintes informações: sexo, idade, tipo de exame realizado, origem das solicitações e absenteísmo no período entre 2014-2016. Resultados: Foram agendados 9890 exames radiográficos, sendo $74,6 \%$ extrabucais e $25,4 \%$ intrabucais. Em relação ao perfil dos pacientes, o sexo feminino representou $61,8 \%$ das marcações, e a faixa etária de 19 a 59 anos de idade foi predominante $(69,1 \%)$. De modo geral, observou-se alto percentual de absenteísmo $(42,1 \%)$, com maiores índices, estatisticamente significantes, relacionados ao sexo feminino $(43,1 \%)$, à faixa etária de 12 a 18 anos $(45,9 \%)$ e à Gerência Distrital Restinga/Extremo Sul $(46,6 \%) \quad(\mathrm{p}<0,05)$. Conclusão: $O$ perfil do usuário do Serviço de Radiologia da FO-UFRGS é predominantemente feminino e adulto, e variáveis como sexo, idade e localização da origem dos encaminhamentos tem correlação com o alto absenteísmo observado.

Palavras-chave: Radiologia. Odontologia. Saúde pública. 


\begin{abstract}
Introduction: The Radiology Service of the Faculty of Dentistry of the Federal University of Rio Grande do Sul (FO-UFRGS) attends the needs of individuals originated from SUS (Public Health System) performing dental radiographies, which are relevant for the diagnosis and treatment planning. The scheduling listing of these exams constitutes a database with potential to generate important indicators for the improvement of the service to the population.

Objective: To evaluate the patient profile, number and referrals origin, and absenteeism in relation to schedules of radiographic examinations performed at the Radiology Service of FO-UFRGS. Materials and methods: Data were collected through the Regulation System (SISREG) of Municipal Health Secretariat of Porto Alegre (SMS-POA) and analyzed the following information: sex, age, type of exam, origin of requests and absenteeism in 2014-2016 period. Results: A total of 9890 radiographic exams were scheduled, $74.6 \%$ of which were extraoral and $25.4 \%$ were intraoral. Regarding the profile of the patients, the female sex represented $61.8 \%$ of the appointments, and the age group from 19 to 59 years of age was predominant $(69.1 \%)$. In general, there was a high percentage of absenteeism $(42.1 \%)$, with the highest indexes, statistically significant, related to female sex (43.1\%), the age group 12 to 18 years old (45.9\%) and the Restinga / Extremo Sul District Management $(46.6 \%)(\mathrm{p}<0,05)$. Conclusion: The user profile of the Radiology Service of FO-UFRGS is predominantly female and adult, and variables such as sex, age and location of referral origin correlate with the high absenteeism observed.
\end{abstract}

Keywords: Radiology. Dentistry. Public health.

\title{
INTRODUÇÃO E JUSTIFICATIVA
}

As doenças bucais são problemas importantes de saúde pública devido à alta prevalência, demanda elevada aos serviços, impacto sobre a vida dos indivíduos em termos de dor, desconforto, limitação, deficiência social e funcional, interferindo sobre a qualidade de vida ${ }^{1}$. A partir desse contexto, mudanças na organização dos serviços odontológicos devem ser feitas, sendo que a rede de atenção básica deve ser responsável pela maioria das ações em saúde bucal ${ }^{2}$. O tratamento de especialidades oferecido nos Centros de Especialidades Odontológicas é uma continuidade do trabalho realizado pela rede de atenção básica ${ }^{3}$.

A fim de estabelecer o diagnóstico de cada enfermidade que atinge a cavidade bucal e a melhor forma de tratá-la, além da entrevista dialogada e do exame clínico, o Cirurgião-dentista pode lançar mão dos exames complementares, que incluem radiografias e outras formas de imagem ${ }^{4}$. As radiografias periapical e panorâmica são as técnicas mais solicitadas entre os exames por imagem de uso odontológico ${ }^{5}$.

Segundo Domingos, Rossato e Bellini ${ }^{6}$ os profissionais da Odontologia devem ter uma compreensão da realidade vivida pela população, norteando seu trabalho de acordo com as questões sociais do país. Para tal, faz-se necessário que sejam conhecidos os fatores individuais associados à utilização do sistema de saúde público e às características de oferta de serviços, incluindo sua finalidade. Dessa maneira, este estudo busca avaliar o perfil do usuário, o número e a origem de solicitações de exames e o absenteísmo no Serviço de Radiologia da FO-UFRGS para o planejamento de estratégias que aprimorem o atendimento de acordo com as necessidades reais desta população, a fim de oferecer um serviço público de qualidade. 


\section{MATERIAIS E MÉTODOS}

Este é um estudo observacional transversal a partir de uma análise retrospectiva dos dados coletados a partir do agendamento de pacientes com necessidade de exames radiográficos realizados no período de 2014-2016 no Serviço de Radiologia da FOUFRGS. Foi submetido e aprovado pela Comissão de Pesquisa da FO-UFRGS (sob o número 31983), e pelo Comitê de Ética em Pesquisa da UFRGS (parecer $n^{\circ} 1.890 .015$ ).

A origem das solicitações dos exames, a faixa etária e o sexo dos pacientes foram avaliados, com as frequências absolutas e relativas, percentualmente, por meio de tabelas e figura. A quantidade de abstenções também foi estudada em relação à amostra total e as características da população.

O banco de dados foi coletado a partir do SISREG (Sistema de Regulação) da Secretaria Municipal de Saúde de Porto Alegre (SMS-POA) que, de acordo com a sua inserção no Serviço de Radiologia, variou o período analisado para os exames radiográficos extra e intrabucais, de outubro de 2014 a dezembro de 2016 e abril de 2015 a dezembro de 2016, respectivamente.

Para a avaliação da idade dos usuários, a amostra foi agrupada em faixas etárias. Os critérios de agrupamento etário foram: crianças (até 11 anos de idade), adolescentes (12 a 18 anos de idade), segundo o que preconiza o Estatuto da Criança e do Adolescente (Lei $n^{\circ} 8.069$ de 14 de julho de 1990) ${ }^{7}$ e, idosos (60 anos ou mais de idade), segundo o Estatuto do Idoso (Lei no 10.741 de 01 de outubro de 2003) ${ }^{8}$, portanto, agrupou-se na categoria adulto o número de pacientes com intervalo de idade entre 19 a 59 anos de idade.

Os encaminhamentos foram analisados por Unidades de origem, entre elas, Unidades Básicas de Saúde (UBS), Estratégias de Saúde da Família (ESF) e Centro de Especialidades Odontológicas (CEO), e posteriormente agrupados por Gerências Distritais para comparação e análise estatística.

A análise de dados se deu por meio manual, com transferência do site do SISREG (Sistema de Regulação) a planilhas do Microsoft Excel com ordem cronológica, por mês. Com essa etapa concluída, os dados foram codificados em números para que pudessem ser analisados no SPSS (Statical Package for Social Sciences). Os testes estatísticos utilizados foram de Qui-quadrado de Pearson $e$ Teste Exato de Ficher.

\section{RESULTADOS}

Foram encontrados 9890 agendamentos para realização de exames radiográficos no Serviço de Radiologia da FO-UFRGS via SISREG da SMS-POA durante o período de outubro de 2014 e dezembro de 2016. Desses agendamentos, 6116 eram de pacientes do sexo feminino e 3774, do masculino. A média de idade foi de 33,7 anos, variando de 0 a 97 anos. Os pacientes foram agrupados por faixas etárias (crianças, adolescentes, adultos e idosos) e foi possível observar que $69,1 \%$ dos agendamentos corresponderam à faixa etária adulta de 19 a 59 anos. Os exames realizados foram classificados quanto ao tipo, extrabucal (panorâmico) e intrabucal 
(periapical e interproximal). Foi observado um total de 7381 exames panorâmicos $(74,6 \%)$ e 2509 exames intra-bucais $(25,4 \%)$. O maior número de encaminhamentos foi da Gerência Distrital Glória/Cruzeiro/Cristal (17,3\%) e o menor foi da Restinga/Extremo Sul (4,2\%) (Tabela 1).

Tabela 1: Número de exames agendados de acordo o perfil dos pacientes atendidos pelo serviço de Radiologia UFRGS (sexo, faixa etária, encaminhamento e tipo de exame)

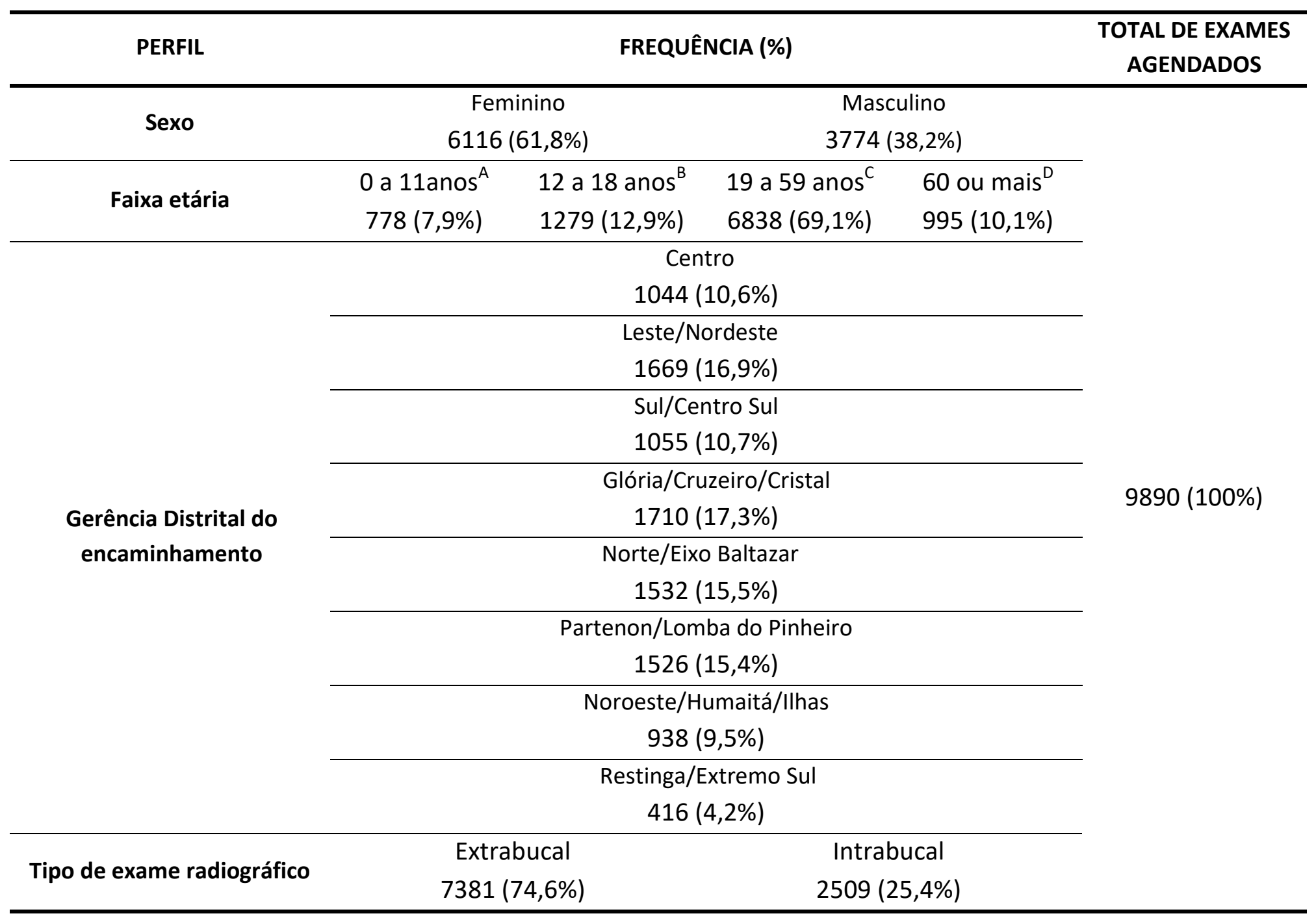

A: crianças, B: adolescentes, C: adultos e D: idosos 
A figura 1 mostra o panorama geral das vagas preenchidas via SISREG por local de origem dos encaminhamentos. Pode-se observar que as Unidades com maior número de encaminhamentos foram: UBS Modelo (5,4\%), CEO Santa Marta $(4,3 \%)$ e ESF Timbaúva I (2,9\%).

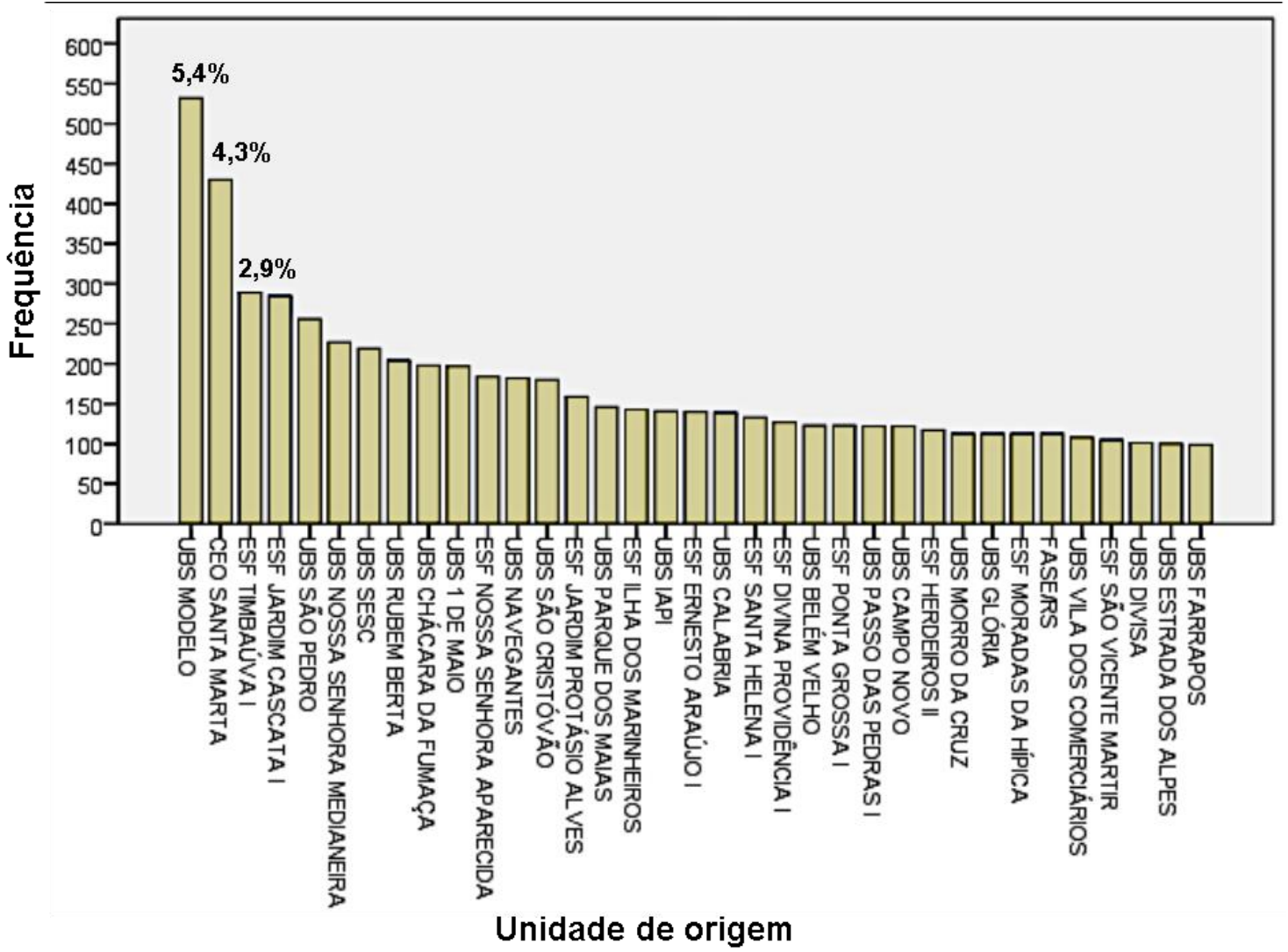

Figure 1 - Número de encaminhamentos de exames ao Serviço de Radiologia FO-UFRGS de acordo com Unidade de origem.

A tabela 2 mostra o absenteísmo nas consultas de exames radiográficos no Serviço de Radiologia segundo o perfil do paciente e a origem dos encaminhamentos. Pode-se observar que pacientes do sexo feminino (43,1\%) apresentam absenteísmo significativamente maior que pacientes do sexo masculino $(40,6 \%)(p<0,05)$. Em relação à faixa etária, as ausências às consultas são significante maiores em pacientes adolescentes $(45,9 \%)(\mathrm{p}<0,05)$. Já frente à origem dos encaminhamentos, verifica-se que a Gerência Distrital Centro apresenta menor índice de absenteísmo (36,7\%), e a Gerência Distrital Restinga/Extremo Sul apresenta o maior índice de absenteísmo $(46,6 \%)$, ambas com diferença estatisticamente significante em relação às demais gerências $(\mathrm{p}<0,05)$. Na análise geral da situação das consultas, referente à presença ou ausência do usuário à consulta, foi encontrado o valor quantitativo de 4166 abstenções, equivalente a 42,1\% dos pacientes agendados.

Tabela 2: Absenteísmo nas consultas de exames radiográficos no Serviço de Radiologia UFRGS, segundo sexo, faixa etária e encaminhamento por gerência distrital. 


\begin{tabular}{|c|c|c|c|c|}
\hline \multirow{2}{*}{ Sexo } & \multicolumn{2}{|c|}{ Feminino } & \multicolumn{2}{|c|}{ Masculino } \\
\hline & \multicolumn{2}{|c|}{$3482 / 2634(43,1 \%)^{a}$} & \multicolumn{2}{|c|}{$2242 / 1533(40,6 \%)^{b}$} \\
\hline \multirow{2}{*}{ Faixa etária } & 0 a 11anos & 12 a 18 anos & 19 a 59 anos & 60 ou mais \\
\hline & $501 / 277(35,6 \%)^{b}$ & $692 / 587(45,9 \%)^{a}$ & $3827 / 3011(44,0 \%) b$ & $704 / 292(29,3 \%)^{b}$ \\
\hline \multirow{16}{*}{$\begin{array}{l}\text { Encaminhamentos } \\
\text { por Gerência Distrital }\end{array}$} & \multicolumn{4}{|c|}{ Centro } \\
\hline & \multicolumn{4}{|c|}{$661 / 383(36,7 \%) \mathrm{Aa}$} \\
\hline & \multicolumn{4}{|c|}{ Leste/Nordeste } \\
\hline & \multicolumn{4}{|c|}{$957 / 712(42,7 \%) A b$} \\
\hline & \multicolumn{4}{|c|}{ Sul/Centro Sul } \\
\hline & \multicolumn{4}{|c|}{$582 / 473(44,8 \%) A b$} \\
\hline & \multicolumn{4}{|c|}{ Glória/Cruzeiro/Cristal } \\
\hline & \multicolumn{4}{|c|}{$963 / 747(43,7 \%) A b$} \\
\hline & \multicolumn{4}{|c|}{ Norte/Eixo Baltazar } \\
\hline & \multicolumn{4}{|c|}{$913 / 619(40,4 \%) \mathrm{Ab}$} \\
\hline & \multicolumn{4}{|c|}{ Partenon/Lomba do Pinheiro } \\
\hline & \multicolumn{4}{|c|}{$875 / 651(42,7 \%) A b$} \\
\hline & \multicolumn{4}{|c|}{ Noroeste/Humaitá/Ilhas } \\
\hline & \multicolumn{4}{|c|}{$550 / 388(41,4 \%) A b$} \\
\hline & \multicolumn{4}{|c|}{ Restinga/Extremo Sul } \\
\hline & \multicolumn{4}{|c|}{$222 / 194(46,6 \%){ }^{\mathrm{Bb}}$} \\
\hline \multirow{2}{*}{ Geral } & \multicolumn{2}{|c|}{ Presença } & \multicolumn{2}{|c|}{ Ausência } \\
\hline & \multicolumn{2}{|c|}{$5724(57,9 \%)$} & \multicolumn{2}{|c|}{$4166(42,1 \%)$} \\
\hline
\end{tabular}

* Percentuais seguidos de diferentes letras maiúsculas e minúsculas, indicam diferença estatisticamente significante ( $p<0,05)$ para o teste exato de Fischer (nível de significância de 5\%).

\section{DISCUSSÃO}

Conhecer o perfil do usuário do Serviço de Radiologia da FO-UFRGS permite compreender as características e o contexto envolvendo essa população, para se refletir acerca dos números de encaminhamentos e absenteísmo correspondente. De forma inédita, este estudo inicia uma sequência de avaliações desse Serviço que colaborará com o aprimoramento e qualidade do atendimento aos pacientes usuários do SUS.

A diferença observada entre os períodos das coletas de dados ocorreu devido aos momentos distintos do início do uso do SISREG para o agendamento dos exames radiográficos intra e extrabucais. Ainda, este trabalho não contabiliza o número de exames radiográficos e sim as vagas disponibilizadas e ocupadas, já que um paciente pode necessitar de diversos exames para a conclusão diagnóstica. Além de um período maior de coleta de dados para os exames extrabucais, cada agendamento nesta categoria corresponde a um exame realizado, o que justifica o resultado amostral maior obtido. Já os exames intrabucais apresentaram um menor período de 
coleta de dados e, neste caso a unidade amostral do paciente não corresponde com o número de radiografias realizadas, pois cada agendamento pode resultar em múltiplas radiografias. Caso o número de exames intrabucais fosse calculado, possivelmente haveria uma simetria entre os tipos de exames, contudo esses dados não foram coletados e avaliados.

Neste estudo, verificou-se que a percentagem majoritária dos agendamentos de exames radiográficos era de pacientes do sexo feminino $(61,8 \%)$, o que corrobora com outros estudos que avaliaram o perfil de usuários da atenção primária. Tortamano et al. ${ }^{9}$ obtiveram um resultado muito próximo, com uma percentagem de $64,6 \%$, e Cassal et al. ${ }^{10}$ verificaram que as mulheres representaram 74,7\% da amostra.

No âmbito da saúde pública, o absenteísmo em consultas tem sido bastante discutido já que traz, de maneira direta, desperdícios de recursos, estruturais e financeiros. No estudo de Miotto et al. ${ }^{11}$ o absenteísmo representou quase $50 \%$ das consultas em Unidades de Saúde da Família. Também, em um estudo em Unidades de Saúde de João Pessoa - PB, Brasil foi possível observar um absenteísmo de 41,2\% ${ }^{12}$ semelhante ao índice encontrado no presente estudo (42,1\%).

A grande maioria dos estudos avaliou o absenteísmo com relação às consultas para tratamento odontológico ${ }^{10,12-17}$, mas poucos avaliaram esta questão com relação ao atendimento para exames radiográficos ${ }^{18-20}$.

Mesmo com a grande demanda da população brasileira ao atendimento odontológico, é notório o alto índice de absenteísmo às consultas. Estudos prévios acerca do absenteísmo em serviços de saúde revelaram uma associação entre o absenteísmo e o grau de escolaridade, condição socioeconômica, transporte, tempo de deslocamento e turnos de trabalho perdidos ${ }^{13,14,21}$. O presente estudo procurou avaliar a associação do absenteísmo com o sexo, a faixa etária e com as Unidades de Saúde que originaram os encaminhamentos.

Em relação ao sexo, o absenteísmo foi mais frequente em agendamentos de pacientes do sexo feminino, o que também foi observado em outro estudo ${ }^{11}$. Esse resultado pode refletir o contexto social da mulher frente as variáveis que se colocam como uma maior barreira a ser ultrapassada em relação a sua presença nas consultas, em comparação com os usuários do sexo masculino, como o cuidado dos filhos e familiares e sua dupla jornada de trabalho. Da mesma forma que, a maior ausência às consultas por pacientes adolescentes, observada neste estudo, pode pressupor a sobreposição de variáreis tanto do paciente como do acompanhante, como a interferência em atividades profissionais e escolares, além do custo duplo de deslocamento.

Como já destacado por Azevedo e Costa, 2010 ${ }^{22}$, limitações geográficas interferem no acesso à Estratégia de Saúde da Família (ESF). No presente estudo verificou-se que duas Gerências Distritais apresentaram índices de absenteísmo significativamente maiores e menores em relação às demais: Restinga/Extremo Sul $(46,6 \%)$ e Centro $(36,7 \%)$, respectivamente. Estes valores têm correspondência direta 
com as suas localizações geográficas, pois as Unidades referidas são às de maior e menor distância em relação ao Serviço de Radiologia da FO-UFRGS.

O atendimento não realizado é uma oportunidade perdida de oferecer assistência, o que destaca a importância da regulação para o sistema de saúde. Essa busca promover a equidade do acesso garantindo a integralidade da assistência, permitindo ajustar a oferta de consultas e procedimentos disponíveis às necessidades imediatas do cidadão, de forma equânime, ordenada, oportuna e racional ${ }^{23}$. A regulação das consultas odontológicas não é inédita, mas é um tema pouco discutido na literatura. Poucos trabalhos foram publicados envolvendo os temas Serviços de Saúde Bucal, Especialidades Odontológicas e Administração em Saúde Pública ${ }^{15-17,24-29}$. Estratégias devem ser elaboradas com o intuito de auxiliar a otimização dos atendimentos organizados pela regulação.

No estudo de avaliação da regulação dos Centros de Especialidades Odontológicas de Joinville $(\mathrm{SC})^{30}$, foi elaborado um protocolo de prioridades e treinamento para uso do sistema SISREG. Para garantir o acesso à consulta especializada dos pacientes regulados e considerados prioritários foram criadas vagas de reserva nos moldes da técnica do overbooking. Foram criadas vagas extras em razão do considerável número de faltas que, para algumas especialidades, às vezes chegava a mais de 50\%. O índice de não comparecimento de usuários às consultas e exames agendados no Sistema Único de Saúde (SUS) é significativamente alto em todo Brasil, de forma geral próximo ou superior a $25 \%^{31}$. Utilizar a técnica do overbooking e a regulação das consultas

podem ser estratégias para a melhor gestão dos serviços públicos de saúde. No entanto, cabe ressaltar que este Serviço de Radiologia está inserido em uma Instituição de Ensino Superior, onde há a participação ativa de alunos de graduação e, consequentemente, o ensino é priorizado. Sendo assim, o trabalho com overbooking não é adotado, pois a sobrecarga pode interferir no processo de aprendizagem. Para respeitar uma curva ascendente de cada etapa etapa, inicia-se, então, as atividades com um número de pacientes que cresce ao longo do período.

Outros estudos devem ser realizados para identificar causas e consequências de falhas do sistema de trabalho e, a partir dessas evidências, estratégias de melhoria e tomadas de decisões podem ser definidas. A busca pela qualidade deve ser permanente quando se trabalha com saúde, tanto para otimização do serviço e recursos, quanto para preocupação com o melhor atendimento ao paciente.

\section{CONCLUSÃO}

Conclui-se que o perfil do usuário do Serviço de Radiologia da FO-UFRGS é predominantemente feminino e adulto, e que variáveis como sexo, idade e localização da origem dos encaminhamentos tem correlação com o alto absenteísmo observado.

\section{AGRADECIMENTOS}


Agradecimento aos Técnicos em Radiologia e alunos de graduação participantes da Extensão em "Rotina em Serviço de Radiologia Odontológica e Imaginologia“ da FO-UFRGS que, com dedicação, atendem aos pacientes encaminhados ao Serviço de Radiologia da mesma Instituição.

\section{REFERÊNCIAS}

1. Baldisserotto J. Declaração de Berlim sobre saúde bucal para comunidades carentes. Rev Divulg. 1995;(10):57-63.

2. Thomas DW, Satterthwaite J, Shepherd JP. Trends in the referral and treatment of new patients at a free emergency dental clinic since 1989. Br Dent J. 1997;182(1):11-4.

3. Brasil. Ministério da Saúde. Centro de Especialidades Odontológicas. [Internet]. Brasília; 2011 [acesso 2016 set 10]. Disponível em: www.saude.gov.br/bucal.

4. Moura LB, Blasco MAP, Damian MF. Exames radiográficos solicitados no atendimento inicial de pacientes em uma Faculdade de Odontologia brasileira. Rev Odontol da UNESP. 2014;43(4):252-7. doi:10.1590/rou.2014.046.

5. Salti L, Whaites EJ. Survey of dental radiographic services in private dental clinics in Damascus, Syria. Dentomaxillofacial Radiol. 2002;31(2):100-5. doi: 10.1038/sj.dmfr.4600676.

6. Domingos PAS, Rossato EM, Bellini A. Levantamento do perfil social, demográfico e econômico de pacientes atendidos na clínica de odontologia do centro universitário de Araraquara - UNIARA. Rev Uniara. 2014 nov;17:37-50.

7. Brasil. Estatuto da criança e do adolescente: Lei federal $n^{\circ} 8069$, de 13 de julho de 1990 - ECA. Brasília, DF [acesso 2018 nov 05]. Disponível em: http://www.planalto.gov.br/ccivil_03/LEIS/L8069.htm

8. Brasil. Estatuto do idoso: lei federal n ${ }^{\circ} 10.741$, de 01 de outubro de 2003. Brasília, DF [acesso 2018 nov 05]. Disponível em: http://www.planalto.gov.br/ccivil_03/LEIS/2003/L10.741.htm

9. Tortamano IP, Leopoldino VD, Borsatti MA, Penha SS, Buscariolo IA, Costa CG, et al. Aspectos epidemiológicos e sociodemográficos do setor de Urgência da Faculdade de Odontologia da Universidade de São Paulo. Rev Pós Grad. 2007; 13 (4):299-306.

10. Cassal JB, Cardozo DD, Bavaresco CS. Perfil dos usuários de urgência odontológica em uma unidade de atenção primária à saúde. Rev. APS, 2011; 14(1):85-92.

11. Miotto, MHMB, Santos, LR, Farias CML. Absenteísmo de usuários às consultas odontológicas em uma unidade de saúde da família. Rev. Bras. Pesq. Saúde, 2015;17(4);121-8.

12. Cavalcanti RP, Cavalcanti JCM, Serrano RMSM, Santana PR. Absenteísmo de consultas especializadas nos sistema de saúde público : relação entre causas e o processo de trabalho de equipes de saúde da família. Rev Tempus Actas Saúde Col, 2010; 63-84.

13. Almeida GL, Garcia LFR, Almeida TL, Bittar TO, Pereira AC. Estudo do perfil 
socioeconômico dos pacientes e os motivos que os levaram a faltar a consultas odontológicas na estratégia de saúde da família em uma distrital de Ribeirão Preto/SP. Ciênc Odontol Bras. 2009; 12(1):77-86.

14. Jandrey CM, Drehmer TM. Absenteísmo no atendimento clínico odontológico: o caso do módulo de serviço comunitário do Centro de Pesquisas em Odontologia Social - UFRGS. Rev Fac Odontol P Alegre. 1999; 40(1):24-8.

15. Pucca GA Jr, Costa JF, Chagas Lde D, Sivestre RM. Oral health policies in Brazil. Braz Oral Res. 2009;23 (Suppl 1):9-16.

16. Gehshan S, Snyder A. Why public policy matters in improving access to dental care. Dent Clin North Am. 2009;53(3):573-89.

17. Burry A. An evolution in progress. The integration of social services and public health dental programs. Ont Dent. 1999;76(2):25-7.

18. Nascimento PBPL, Costa SM, Rímulo ALM, Almeida AMR. Serviço de radiologia odontológica em municípios da região Centro- Oeste do Estado de Minas Gerais , Brasil. Rev Odontológica do Bras Cent. 2014;23(64):30-4.

19. Lira-Júnior R, Wanderley Cavalcanti Y, de Fátima Dantas de Almeida L, Oliveira de Sales MA. Panorama da radiologia odontológica no Brasil: disponibilidade de aparelhos e produção ambulatorial de radiografias. Rev Cubana Estomatol. 2012;49(3):223-31.

20. Atchison KA, White SC, Flack VF, Hewlett ER. Assessing the FDA guidelines for ordering dental radiographs. J Am Dent Assoc. 1995;126(10):1372-83.

21. Rohr RIT, Barcellos AL. As barreiras de acesso para os serviços odontológicos. UFES Rev Odontol. 2008; 10(3):37-41.

22. Azevedo ALM, Costa AM. The narrow entrance door of Brazil's National Health System (SUS): an evaluation of accessibility in the Family Health Strategy. Interface - Comunic., Saude, Educ. 2010 Out-Dez; 14(35):797-810.

23. Brasil. Portaria no 399, de 22 de fevereiro de 2006. Divulga o pacto pela saúde 2006 - Consolidação do SUS e aprova as diretrizes operacionais do referido pacto. Diário Oficial [da] república Federativa do Brasil, Brasília, DF, 23 fev. 2006. p. 43-5.

24. Byck GR, Cooksey JA, Russinof H. Safety-net dental clinics. J Am Dent Assoc. 2005;136(7):1013-21.

25. Kuthy RA, Odom JG. Local dental programs: a descriptive assessment of funding and activities. J Public Health Dent. 1988;48(1):36-42.

26. Hastreiter RJ, Lambo AM, Anderson HA. Public health officials' perceptions of dental caries preventive methods. J Public Health Dent. 1985;45(3):180-6.

27. Evans CA Jr. A national survey of dental public health services in local health departments: a report of findings. J Public Health Dent. 1984;44(3):112-9.

28. The University of North Carolina at Chapel Hill. Department of Continuing Education. School of Public Health. Behavioral objectives for dental public health. J Public Health Dent. 1978 Winter;38(1):99-108.

29. Wisan JM, Chilton NW. Studies in dental public health administration; administrative factors in a dental treatment program as measured by lost time analysis. J Am Dent Assoc. 1948;36(1):39-52. 
30. Abuabara A. Avaliação do primeiro ano de regulação dos procedimentos dos Centros de Especialidades Odontológicas de Joinville (SC). Rev Oficial do Núcleo de Estudos da Saúde do Adolescente.[Internet]. [acesso 2017 jul 10]. Disponível em: http://www.adolescenciaesaude.com/detalhe_artigo.asp?id=611

31. Oleskovicz M, Oliva FL, Grisi CC, Lima AC, Custódio I. Overbooking in an outpatient healthcare facility in the Brazilian Unified National Health Sist Cad Saude Publica. 2014;30:1009-1. 